\title{
A Case of Burkitt-Like Lymphoma With 11q Aberration With HIV Infection in East Asia and Literature Review
}

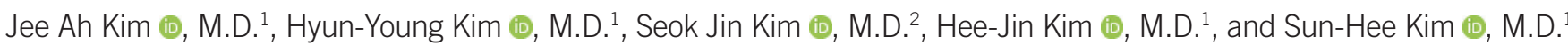 \\ ${ }^{1}$ Department of Laboratory Medicine and Genetics and ${ }^{2}$ Division of Hematology-Oncology, Department of Medicine, Samsung Medical Center, \\ Sungkyunkwan University School of Medicine, Seoul, Korea
}

\section{Dear Editor,}

Burkitt-like lymphoma with $11 q$ aberration (BLL-11q) is a new provisional classification in the revised fourth edition of WHO classification of lymphomas that resembles Burkitt lymphoma (BL) morphologically and phenotypically, but has unique features, including gains in 11q23.2-23.3 and losses of 11q24.1qter, with no MYC translocation [1]. BLL-11q predominantly occurs in young adult males, and characteristic cytogenetic features have been frequently observed in post-transplant patients [2-4]. However, the association between BLL-11q and immune deficiency remains unclear due to the limited number of cases [2, 4-6]. We report a case of BLL-11q with HIV co-infection in East Asia, and review previous cases of BLL-11q with immunodeficiency. The Institutional Review Board of Samsung Medical Center, Seoul, Korea, approved this study (IRB No: SMC-202005-125) and waived the need for informed consent.

A 23-year-old male who was previously healthy was admitted to Samsung Medical Center in January 2020 for investigation of a palpable mass on the left axilla and back pain. Axillary lymphnode biopsy was positive for CD10, BCL6, and Ki-67 (>90\%), and negative for BCL2, CD3, and MUM-1 (IRF-4). FISH was negative for $B C L 2$ or $B C L 6$ rearrangement. Bone marrow (BM) aspirate and section revealed an increase in medium- to largesized lymphoma cells (Fig. 1A and 1B). Flow cytometry was per- formed on BM aspirates. After staining with monoclonal antibodies against B-cell associated antigens, data acquisition and analysis were performed using the FACSLyric flow cytometer (Becton Dickinson, San Jose, CA, USA) and Kaluza flow cytometry analysis software (Beckman Coulter Inc., Indianapolis, IN, USA), respectively. Lymphoma cells were positive for CD10, CD19, CD20, and CCD79a, moderately positive for CCD22, and negative for CD34, terminal deoxynucleotidyl transferase (TdT), CD5, and kappa/lambda surface immunoglobulins. Immunohistochemical staining was performed on formalin-fixed, paraffin-embedded $B M$ sections using monoclonal antibodies against CD3, CD20, CD34, and TdT (DAKO, Santa Clara, CA, USA), and showed lymphoma cells diffusely positive for CD2O (Fig. 1C) and reactive T cells positive for CD3 (Fig. 1D). Epstein-Barr virus in-situ hybridization was negative. Chromosomal analysis revealed 46,XY,der (11)dup(11)(q24q13)del(q24)[18]/46,XY[2] (Fig. 1E). FISH analysis using LSI IGH/MYC/CEP8 Tri-Color Dual Fusion probe (Vysis, Abbott Park, IL, USA), LSI KMT2A (MLL) Dual-Color Break Apart probe (Vysis), and TelVysion 11q SpectrumOrange probe (Vysis) indicated the presence of $11 \mathrm{q}$ aberrations (Fig. 1F, 1G), with no MYC rearrangement.

Chromosomal microarray analysis was conducted using GeneChip System 3000Dx v.2 (Thermo Fisher Scientific, Carlsbad, CA, USA) and a CytoScan Dx Assay (Thermo Fisher Scientific).
Received: September 15, 2020

Revision received: November 30, 2020

Accepted: May 7, 2021

Corresponding author: Sun-Hee Kim, M.D.

Department of Laboratory Medicine and Genetics, Samsung Medical Center, Sungkyunkwan University School of Medicine, 81 Irwon-ro, Gangnam-gu, Seoul 06351, Korea

Tel: +82-2-3410-2704, Fax: +82-2-3410-2719

E-mail: sunnyhk@skku.edu 

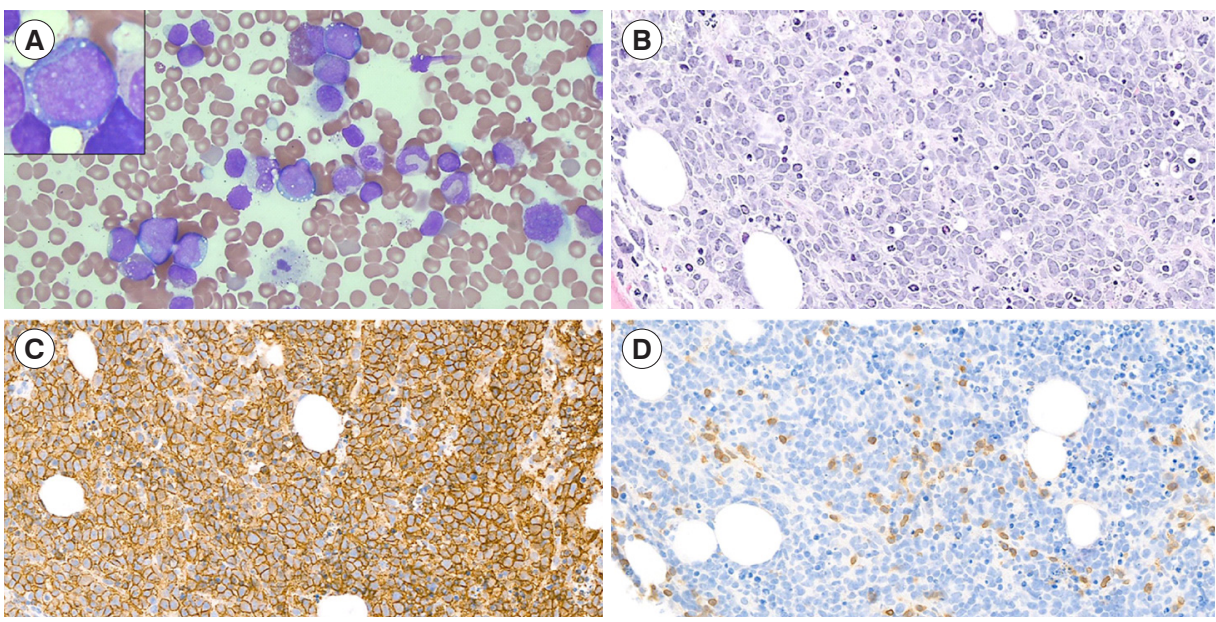

E
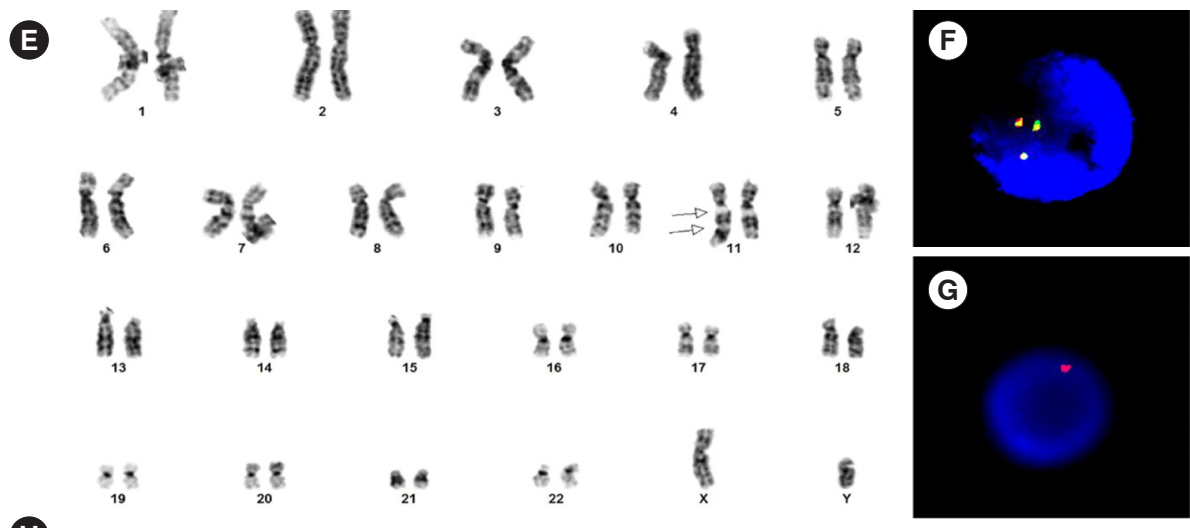

$\boldsymbol{H}$

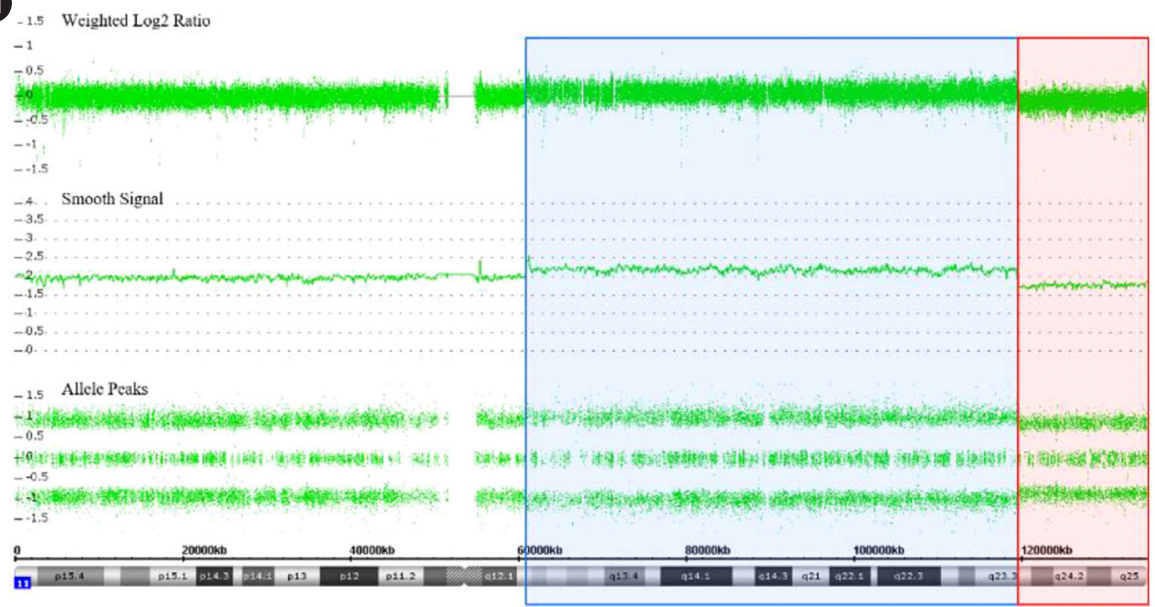

Fig. 1. Bone marrow findings in Burkitt-like lymphoma with 11q aberration. (A) Bone marrow aspirate smear revealed medium- to largesized lymphoma cells with finely clumped chromatin, variably prominent nucleoli, moderate amounts of deeply basophilic cytoplasm, with or without lipid vacuoles (Wright-Giemsa stain, $\times 400$ and $\times 1,000$ ). (B) Bone marrow biopsy section revealed diffuse infiltration of lymphoma cells composed of medium- to large-sized cells ( $H$ \& E stain, $\times 200$ ). On immunohistochemical staining, (C) lymphoma cells were diffusely positive for CD20, and (D) reactive T cells were positive for CD3 ( $\times 200)$. (E) Chromosome analysis showed an abnormal chromosome 11, including inverted duplication of the part of the long arm between 11q24 and 11q13 and terminal deletion of the long arm from 11q24 to 11ter (arrow). On interphase fluorescence in-situ hybridization, (F) KMT2A (MLL) Dual-Color Break Apart probe (Vysis) showed three green/orange (yellow) fusion signals indicating a gain of 11q23.3, and (G) Telomere 11q SpectrumOrange probe (Vysis) showed a single orange signal, indicating a loss of 11q24. (H) Chromosomal microarray analysis showed a copy-number gain of 11q12.2 q23.3 (blue) followed by an adjacent distal loss of 11 q23.3 q25 (red). 


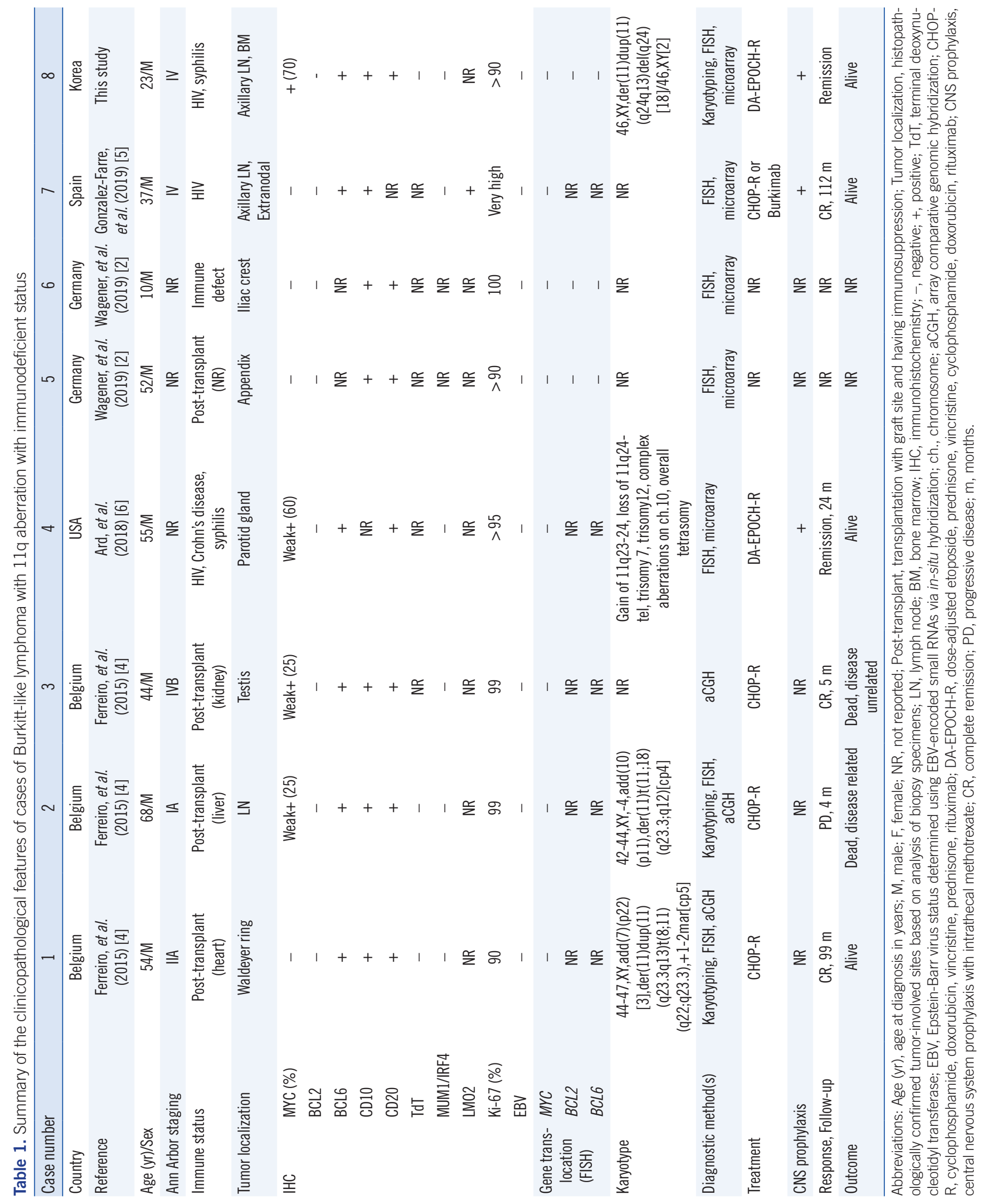


The results showed a $58.5 \mathrm{Mb}$ copy number gain on 11q12.2q23.3 (chr11:60,902,421-119,465,669) and a 15.4 Mb copy number loss on 11q23.3q25 (chr11:119,465,715-134,938,470) (Fig. $1 \mathrm{H})$. Targeted next-generation sequencing using a NextSeq 550Dx instrument (Illumina, San Diego, CA, USA) with IDT xGen predesigned/custom probes (Integrated DNA Technologies, Coralville, IA, USA) of 90 genes linked with B-cell lymphoma identified variants in GNA13, ARID1A, KMT2D, TP53, PTEN, and BRAF. Additional workups showed positive results for both screening and confirmative tests for HIV infection, with a CD4+ T-cell count of 228/ $\mathrm{LL}$. Finally, the patient was diagnosed as having stage IV BLL-11q with HIV and late latent syphilis co-infection. Subsequently, he was treated with dose-adjusted etoposide, prednisone, vincristine, cyclophosphamide, doxorubicin, and rituximab (DA-EPOCH-R) for B-cell lymphoma.

After five cycles of chemotherapy over four months, together with markedly improved imaging findings and no involvement of lymphoma in the follow-up BM study, the patient achieved remission. After four months of antiretroviral therapy, HIV RNA was below the detection limit, with an increase in $C D 4+T$ cells by 316 cells $/ \mu \mathrm{L}$.

To the best of our knowledge, this is the first case of BLL-11q accompanied by HIV infection in East Asia. We identified the 11q aberrations by cytogenetic studies, suggesting that these technical approaches may be useful for the diagnosis of BLL$11 \mathrm{q}$.

Even though flow cytometry indicated mature B-cell lymphoma in our case, there was no expression of surface immunoglobulins. Surface immunoglobulin light chain-negative B-cell nonHodgkin lymphomas (NHLs) as well as lymphomas in HIV-infected patients tend to be clinically aggressive [6-8]. However, our patient had a good performance status with an Eastern Cooperative Oncology Group performance score of 0 , with no systemic symptoms. These features may support the favorable outcome of BLL-11q.

As previously reported in BLL-11q [5], we detected variants in GNA13, ARID1A, and KMT2D, which are frequently found in diffuse large B-cell lymphomas (DLBCL), but not in BL. This corroborates that BLL-11q has similar clinicopathological and immunophenotypic features as BL, but different molecular features. Thus, the hypothesis that BLL-11q is a discrete entity of high-grade B-cell lymphoma (HGBCL) rather than a subdivided entity belonging to $\mathrm{BL}$ needs to be considered.

The relationship between BLL-11q and immunocompromised conditions has not been fully evaluated [5]. A literature review revealed seven cases of BLL-11q in immunodeficient settings
(Table 1). These cases occurred strictly in males, as women are protected by estrogens against the proliferation of B-cell NHLs [9]. Interestingly, all cases with HIV infection, including our patient, showed either an advanced tumor stage or an aggressive clinical course, but had a good response to chemotherapy.

The optimal therapy for BLL-11q remains controversial. Despite the poor prognostic factors in our patient, such as advancedstage disease, BM involvement, and low absolute lymphocyte count, low-intensity chemotherapy (DA-EPOCH-R) was effective. These outcomes strengthen the favorable prognosis of BLL$11 q$ and suggest consideration of dose-reduced therapy for this subgroup of lymphomas [3, 10].

Our study results suggest that detailed histopathological and cytogenetic studies are especially important in young patients with underlying immunodeficiency who exhibit morphologies of $B L, D L B C L$, and HGBCL. The favorable prognosis of BLL-11q emphasizes the significance of accurate diagnosis in such cases. Further research will be needed to elucidate the molecular pathogenesis of and optimal therapeutic strategies for this malignancy.

\section{ACKNOWLEDGEMENTS}

None.

\section{AUTHOR CONTRIBUTIONS}

Kim JA wrote the manuscript. Kim SJ managed the patient and provided the clinical information. Kim HY, Kim HJ, and Kim SH contributed to the interpretation of the results and revision of the manuscript.

\section{CONFLICT OF INTEREST}

None declared.

\section{RESEARCH FUNDING}

None declared.

\section{ORCID}

Jee Ah Kim Hyun-Young Kim

Seok Jin Kim

Hee-Jin Kim

Sun-Hee Kim https://orcid.org/0000-0002-7011-1537 https://orcid.org/0000-0003-0553-7096 https://orcid.org/0000-0002-2776-4401 https://orcid.org/0000-0003-3741-4613 https://orcid.org/0000-0002-7542-5551 


\section{REFERENCES}

1. Swerdlow SH, Campo E, et al. eds. WHO classification of tumours of haematopoietic and lymphoid tissues. 4th ed. Lyon: IARC Press, 2017; 334.

2. Wagener R, Seufert J, Raimondi F, Bens S, Kleinheinz K, Nagel I, et al. The mutational landscape of Burkitt-like lymphoma with 11q aberration is distinct from that of Burkitt lymphoma. Blood 2019;133:962-6.

3. Au-Yeung RKH, Arias Padilla L, Zimmermann M, Oschlies I, Siebert R, Woessmann W, et al. Experience with provisional WHO-entities large Bcell lymphoma with IRF4-rearrangement and Burkitt-like lymphoma with $11 \mathrm{q}$ aberration in paediatric patients of the NHL-BFM group. $\mathrm{Br} \mathrm{J}$ Haematol 2020;190:753-63.

4. Ferreiro JF, Morscio J, Dierickx D, Marcelis L, Verhoef G, Vandenberghe $\mathrm{P}$, et al. Post-transplant molecularly defined Burkitt lymphomas are frequently MYC-negative and characterized by the 11q-gain/loss pattern. Haematologica 2015;100:e275-9.

5. Gonzalez-Farre B, Ramis-Zaldivar JE, Salmeron-Villalobos J, Balagué O, Celis V, Verdu-Amoros J, et al. Burkitt-like lymphoma with 11q aberra- tion: a germinal center-derived lymphoma genetically unrelated to Burkitt lymphoma. Haematologica 2019;104:1822-9.

6. Ard KL, Kelly HR, Gandhi RT, Louissaint A, Jr. Case 9-2018: A 55-yearold man with HIV infection and a mass on the right side of the face. $\mathrm{N}$ Engl J Med 2018;378:1143-52.

7. Coté TR, Biggar RJ, Rosenberg PS, Devesa SS, Percy C, Yellin FJ, et al. Non-Hodgkin's lymphoma among people with AIDS: incidence, presentation and public health burden. AIDS/Cancer Study Group. Int J Cancer 1997;73:645-50.

8. Matsushita H, Nakamura N, Tanaka Y, Ohgiya D, Tanaka Y, Damdinsuren A, et al. Clinical and pathological features of B-cell non-Hodgkin lymphomas lacking the surface expression of immunoglobulin light chains. Clin Chem Lab Med 2012;50:1665-70.

9. Horesh $\mathrm{N}$ and Horowitz NA. Does gender matter in non-hodgkin lymphoma? Differences in epidemiology, clinical behavior, and therapy. Rambam Maimonides Med J 2014;5:e0038.

10. Gastwirt JP and Roschewski M. Management of adults with Burkitt lymphoma. Clin Adv Hematol Oncol 2018;16:812-22. 